\title{
Microstructure-Based Interfacial Tuning Mechanism of Capacitive Pressure Sensors for Electronic Skin
}

\author{
Weili Deng, ${ }^{1}$ Xinjie Huang, ${ }^{2}$ Wenjun Chu, ${ }^{2}$ Yueqi Chen, ${ }^{2}$ Lin Mao, ${ }^{2}$ \\ Qi Tang, ${ }^{2}$ and Weiqing Yang ${ }^{1}$ \\ ${ }^{1}$ Key Laboratory of Advanced Technologies of Materials (Ministry of Education), School of Materials Science and Engineering, \\ Southwest Jiaotong University, Chengdu 610031, China \\ ${ }^{2}$ School of Mechanical Engineering, Southwest Jiaotong University, Chengdu 610031, China
}

Correspondence should be addressed to Weiqing Yang; wqyang@swjtu.edu.cn

Received 30 November 2015; Revised 17 January 2016; Accepted 20 January 2016

Academic Editor: Stefania Campopiano

Copyright (C) 2016 Weili Deng et al. This is an open access article distributed under the Creative Commons Attribution License, which permits unrestricted use, distribution, and reproduction in any medium, provided the original work is properly cited.

\begin{abstract}
In order to investigate the interfacial tuning mechanism of electronic skin (e-skin), several models of the capacitive pressure sensors (CPS) with different microstructures and several sizes of microstructures are constructed through finite element analysis method. The simulative pressure response, the sensitivity, and the linearity of the designed CPS show that the sensor with micropyramids has the best performance in all the designed models. The corresponding theoretically predicted sensitivity is as high as $6.3 \times 10^{-7} \mathrm{fF} / \mathrm{Pa}$, which is about 49 times higher than that without any microstructure. Additionally, these further simulative results show that the smaller the ratios of $L / H$ of pyramid, the better the sensitivity but the worse the linearity. When the ratio of $L / H$ of pyramid is about $\sqrt{2}$, the sensitivity and the linearity could reach a balance point. The simulative results evidently provide the important theoretically directive significance for the further development of $e$-skin.
\end{abstract}

\section{Introduction}

Electronic skin (e-skin) is usually referred to as "the new flexible wearable tactile biomimetic sensor." The rapid progress of an $e$-skin with human-like sensory capabilities has been made by the possibility of such large, multisensory surfaces being highly applicable for autonomous artificial intelligence, medical diagnostics, and replacement prosthetic devices capable of providing the same level of sensory perception as the organic equivalent [1]. The working mechanisms of human machine interfacing for $e$-skin reported mainly focused on the piezoresistivity $[2,3]$, capacitance $[4,5]$, piezoelectricity $[6,7]$, optics $[8,9]$, and so on. Among them, capacitive sensors based $e$-skin simplifies device design and analysis owing to the simple governing equation $C=\varepsilon_{0} \varepsilon_{r} S / \delta$ [10], where $\varepsilon_{0}$ is the free space permittivity, $\varepsilon_{r}$ is the relative permittivity, $S$ is the opposite area, and $\delta$ is the distance between electrodes. Moreover, capacitive sensors for tactile sensing of $e$-skin have demonstrated high strain sensitivity, compatibility with static force measurement, and low power consumption [10-12]. After the first monolithic capacitive pressure sensor was designed for medical-research applications in 1980 [13], silicon solution tablets technology and etch-stop technique were put forward to make the capacitive sensor [14]. Afterwards, the CPS with different microstructures had been deeply studied due to the introduction of MEMS (microelectromechanical systems) technology [1518]. Ji et al. [19] made the capacitive pressure sensor by a combination of a standard CMOS (Complementary Metal Oxide Semiconductors) process and bulk micromachining technology. Tian and coworkers [20] designed a flexible capacitive pressure sensor with pyramids in 2010 and they analyzed its response time by experimental studies. These results showed that the pyramid structure had the advantage of short response times, evidently demonstrating the superbly capably tuning action of microstructure interface between human skin and $e$-skin on the performance of the CPS. Therefore, of important significance for the great improvement of pressure sensor performance is making clear the interfacial microstructure-tuning mechanism. However, 
there is scarcely any report to theoretically and systematically investigate the interfacial tuning mechanism of $e$-skin to date. Here, we employ the traditional COMSOL Multiphysics models using finite element analysis method [2124] to construct four different microstructures and several sizes of microstructures for the detailed study of interfacial microstructure-tuning mechanism between human skin and PVDF (polyvinylidene fluoride). The theoretical model of the CPS with microstructure decorated is proposed. And the pressure response, the sensitivity, and the nonlinear error of the designed sensors are analyzed. Both the results from theoretical analysis and simulation calculation could be used as theoretical guidance in $e$-skin processing.

\section{System Design and Characterization}

As we know, the capacitance of parallel plate capacitors is

$$
C=\frac{\varepsilon S}{\delta}=\frac{\varepsilon_{0} \varepsilon_{r} S}{\delta},
$$

where $\varepsilon$ is the dielectric permittivity of dielectric medium; $\varepsilon_{0}$ is the vacuum dielectric constant; $\varepsilon_{r}$ is the relative dielectric constant; $S$ is the opposite area of the electrodes; $\delta$ is the distance between the electrodes. According to (1), $S$ and $\delta$ are the two important factors to tune the sensitivity of the CPS. Experimentally, the microstructures surface modifications of the middle dielectric layers were usually employed to effectively control the opposite area $S$ and the distance $\delta$ of electrodes to improve the performance of the CPS. Herein, in order to obtain their theoretical mechanisms, we have designed the middle layers with four different microstructures to get a systematic insight of microstructures surface modifications into the tuning process of the CPS. The sketch of the CPS for $e$-skin is shown in Figure 1.

In this model, both the top and bottom electrodes are the copper thin film materials and the dielectric material is PVDF with excellent dielectric property and flexibility [25]. The substrate is PET (polyethylene terephthalate), which is transparent, and has excellent physical and mechanical properties and electrical insulating properties [5]. Its initial capacity value should be demonstrated as

$$
C_{0}=\frac{\varepsilon S}{\delta_{0}},
$$

in which $\delta_{0}$ is the initial distance between the electrodes and $C_{0}$ is the initial capacitance. When the plate moves,

$$
\Delta C=\frac{\varepsilon S}{\delta_{0}-\Delta \delta}-\frac{\varepsilon S}{\delta_{0}}=\frac{\varepsilon S}{\delta_{0}} \cdot \frac{\Delta \delta}{\delta_{0}-\Delta \delta}=C_{0} \frac{\Delta \delta}{\delta_{0}-\Delta \delta},
$$

where $\Delta \delta$ is the variation of the plate spacing. The relative variation of capacitance can be presented as

$$
\frac{\Delta C}{C_{0}}=\frac{\Delta \delta}{\delta_{0}-\Delta \delta}=\frac{\Delta \delta / \delta_{0}}{1-\Delta \delta / \delta_{0}} .
$$

The Taylor expansion of (4) is shown as

$$
\frac{\Delta C}{C_{0}}=\frac{\Delta \delta}{\delta_{0}}\left[1+\frac{\Delta \delta}{\delta_{0}}+\left(\frac{\Delta \delta}{\delta_{0}}\right)^{2}+\left(\frac{\Delta \delta}{\delta_{0}}\right)^{3}+\cdots\right] .
$$

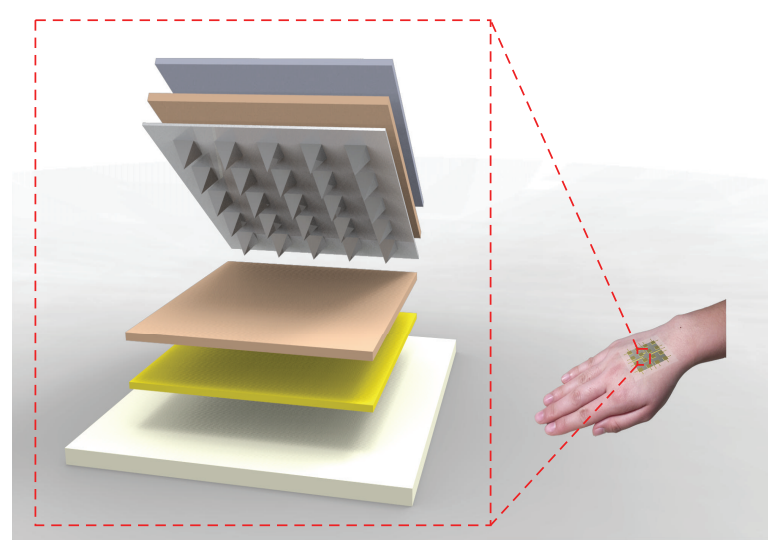

Polyimide Copper

Polyacrylic resin

PVDF

FIGURE 1: The sketch of the CPS for $e$-skin. The sensor has six layers, namely, polyimide, copper, PVDF, copper, polyacrylic resin, and PET. The upper end uses polyimide material as the cover layer; the two pieces of copper used as the electrode, polyacrylic resin as the adhesive coating, and all the devices put on the PET base.

When $\Delta \delta / \delta_{0} \ll 1$, the result (omitting the high-order) can be simplified as

$$
\frac{\Delta C}{C_{0}} \approx \frac{\Delta \delta}{\delta_{0}} .
$$

The sensitivity $K[26]$ can be defined as

$$
K=\frac{\Delta C}{\Delta \delta}=\frac{C_{0}}{\delta_{0}}=\frac{\varepsilon S}{\delta_{0}^{2}} \propto \frac{1}{\delta_{0}^{2}} .
$$

Nonlinear error $r[27,28]$, namely, linearity, is usually measured in terms of a deviation from an ideal straight line and it is typically expressed in terms of percent of full scale, which can be explained as

$$
r=\frac{\Delta C_{\max }}{C_{0}} \times 100 \%=\frac{\Delta \delta_{\max }}{\delta_{0}} \times 100 \% .
$$

\section{Results and Discussion}

In this paper, the middle layers with four microstructures for CPS were designed to investigate the interfacial microstructure-tuning mechanisms of surface modification. As shown in Figure 2, the sketches of these structures are, respectively, flat panel (Figure 2(a)), cuboids (Figure 2(b)), cylinders (Figure 2(c)), and pyramids (Figure 2(d)).

In order to investigate the effect of middle dielectric layer microstructure on the sensitivity of pressure sensor, all hemline lengths $(L)$ of the above microstructures are identically fixed to be $10 \mu \mathrm{m}$, and their heights $(H)$ are designed to the same size of about $7.07 \mu \mathrm{m}$. The corresponding ratios of $L / H$ are controlled to be about $\sqrt{2}$. As shown in Figure 2, under the same pressure of $1 \mathrm{MPa}$, the simulative pressure responses 


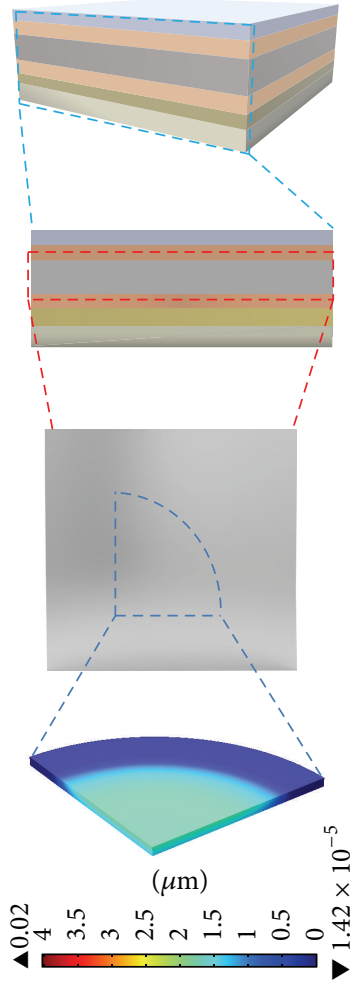

(a)

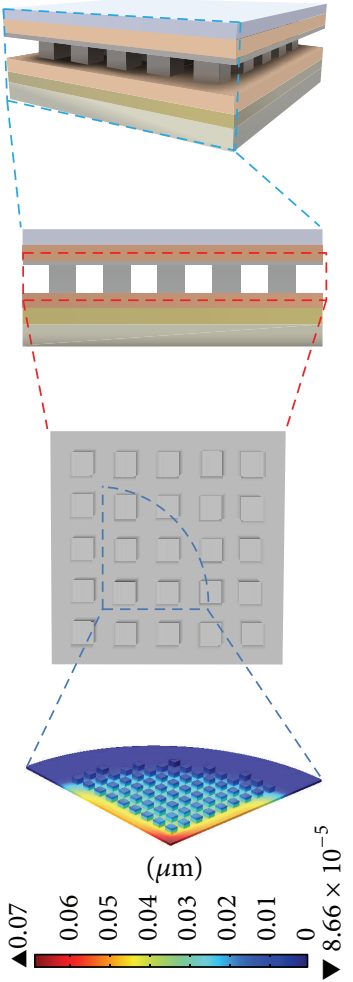

(b)

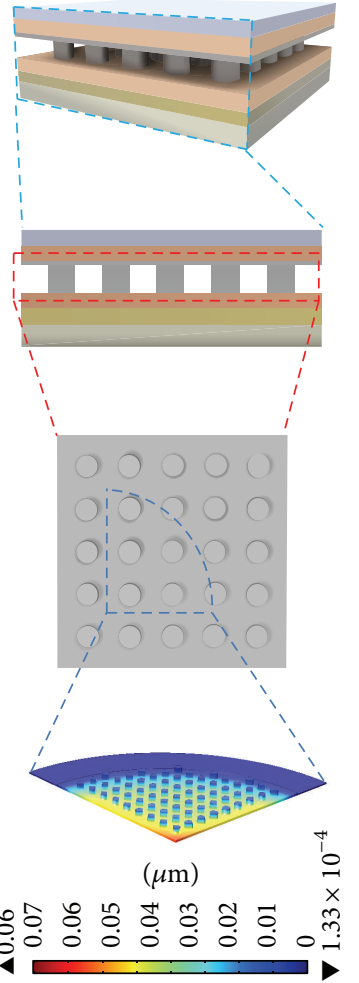

(c)

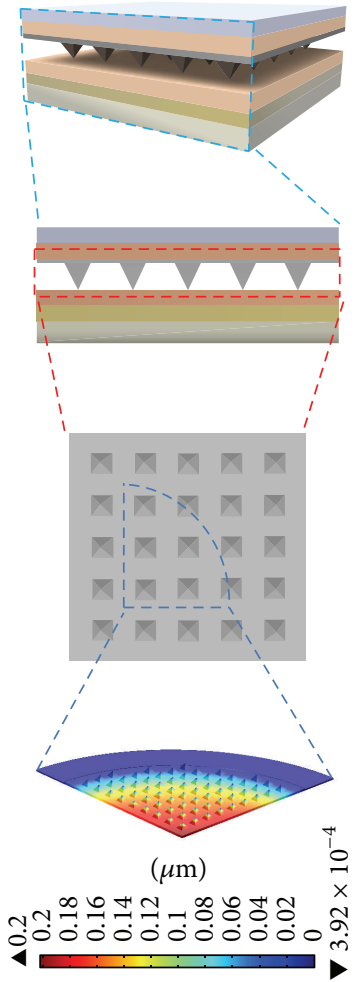

(d)

FIGURE 2: Different profile structures of the CPS. (a) The flat panel, (b) the cuboids, (c) the cylinders, and (d) the pyramids. The 3D diagrams of the CPS, the cross-sectional view of sensors, the vertical view of the functional layer of the sensor, and the deformations of the functional layer are listed in the figure from top to bottom.

TABLE 1: Sensitivity and nonlinear error of the CPS with different microstructures.

\begin{tabular}{lcc}
\hline Structure & Sensitivity $K(\mathrm{fF} / \mathrm{Pa})$ & Nonlinear error $r$ \\
\hline Flat panel & $1.28273 E-8$ & $0.001646 \%$ \\
Cuboids & $8.05555 E-8$ & $0.000681 \%$ \\
Cylinders & $1.00342 E-7$ & $0.000476 \%$ \\
Pyramids & $6.29792 E-7$ & $0.008034 \%$ \\
\hline
\end{tabular}

of CPS evidently show that the sensitivity $K$ of the CPS with microstructures should be better than that without any microstructure, and that of pyramids-based CPS is obviously the best one among four microstructures. Furthermore, the detailed characteristic analyses of the above four structures within the ambient pressure range of $0 \sim 1 \mathrm{MPa}$ through the finite element software COMSOL Multiphysics are shown in Figure 3.

Figures 3(a)-3(d) represent the obvious deformation of the CPS with the increasing pressure. The central areas of CPS have the largest deformation in each model. The capacitance pressure characteristic curves of all the models are shown in Figure 3(e). The comparisons of the sensors' sensitivity $K$ and nonlinear error $r$ between different microstructures are shown in Figure 3(f) and Table 1, showing the sensitivities of pyramids-based and flat panel pressure sensors are
$6.3 \times 10^{-7}$ and $1.28 \times 10^{-8} \mathrm{fF} / \mathrm{Pa}$, respectively. That is, the former is about 49 times higher than the latter, evidently revealing the excellent capacity for microstructure-control of middle dielectric layer to tune the sensitivity of CPS. From Figure 3(f), the nonlinear error $r$ of pyramids is greater than that of other models but still less than $1 \%$, evidently showing the designed CPS has very good linearity. This result is consistent with the discussion of the literature [26]. This can be explained by considering the stress distribution of the different geometrical shapes. In a flat panel structure, the stress distribution is fairly constant throughout the height of the panel to the force contact area. However, when the shape changes from the flat panel to cuboids, cylinders, and pyramids, the stress distribution is nonuniform and is concentrated at the pointed tips due to the smaller contact area, rather than the broad bases of the structures. Thus, the pyramid tips compress more, resulting in the higher mechanical deformations at the top. Therefore, the pyramids shapes are more sensitive.

In order to further study the performance of sensor decorated with pyramids, a series of simulations were performed with different $L$ of pyramids; the sketches and simulation results of the models are shown in Figure 4.

In the simulation, $L$ changes from $8 \mu \mathrm{m}$ to $12 \mu \mathrm{m}$ with constant height $(H=7.07 \mu \mathrm{m})$. The pressure responses of the CPS with different hemline lengths of pyramids are shown in 

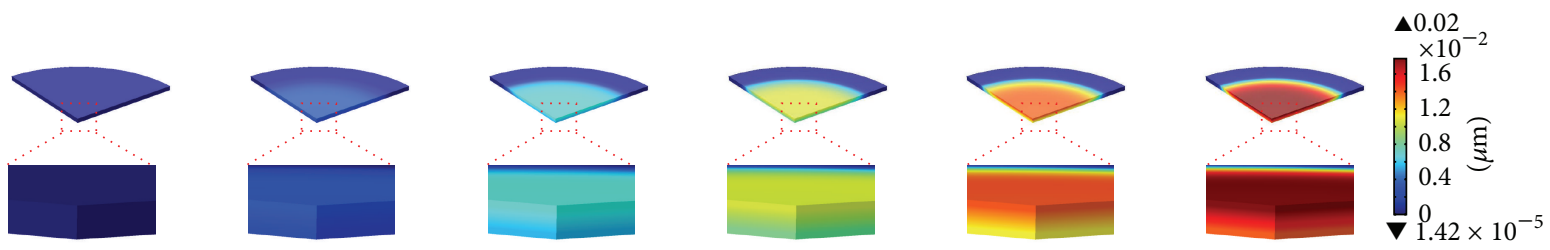

(a)
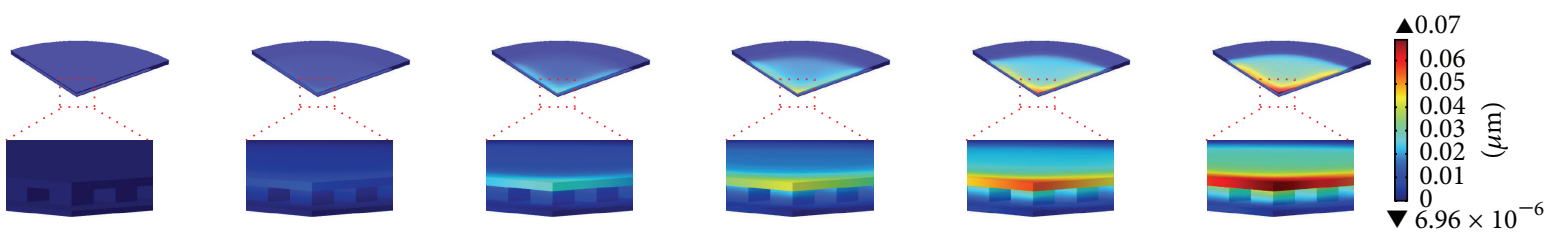

(b)
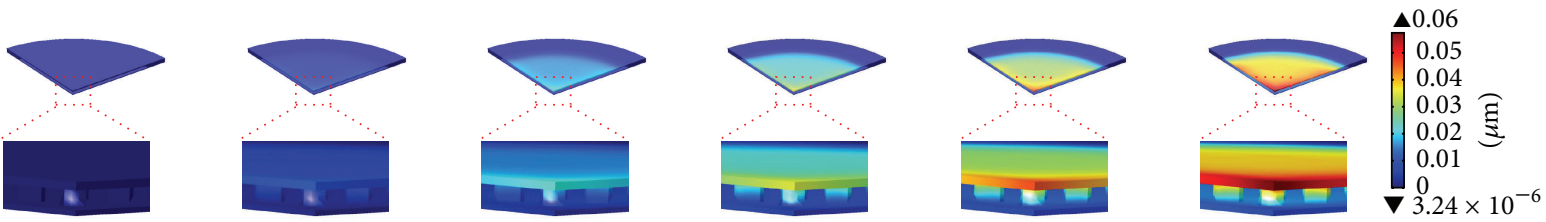

(c)

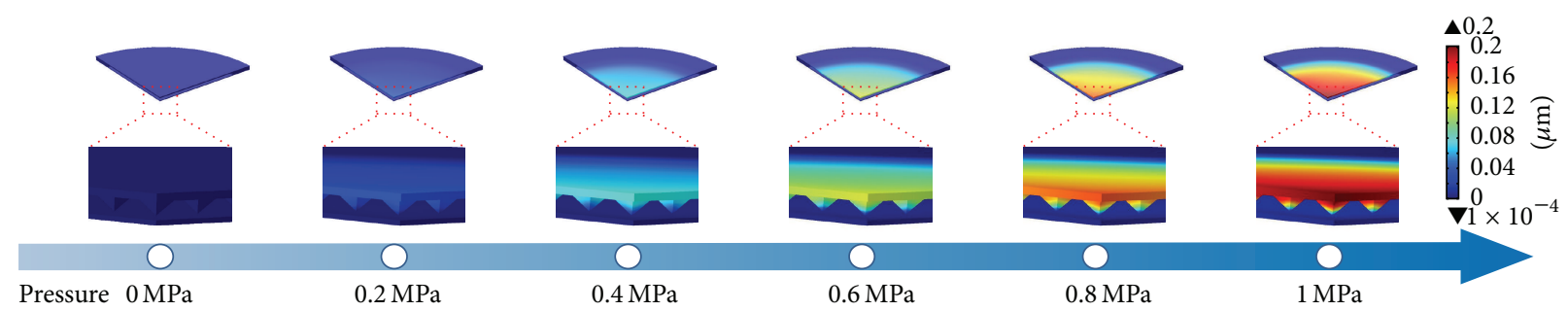

(d)
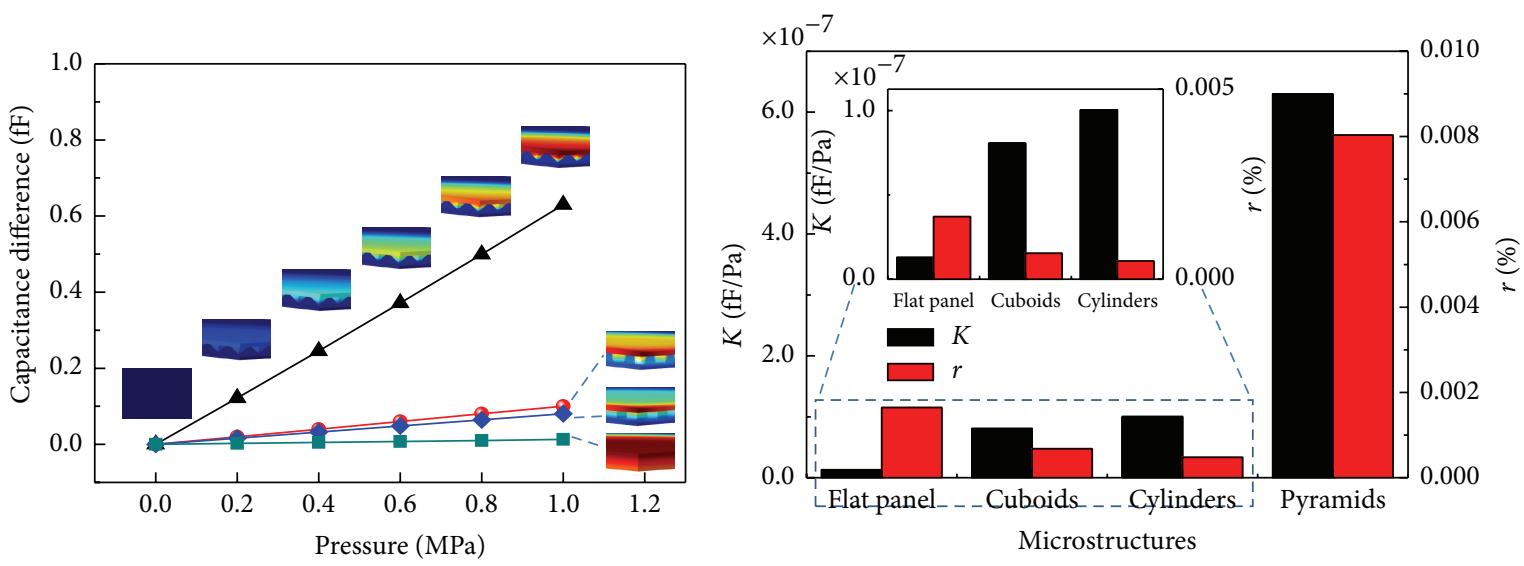

$$
\underset{- \text { - Pyramid }}{- \text { Cylinder }}
$$

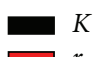

(e)

(f)

FIGURE 3: The pressure responses of the CPS with different microstructures. The deformations of the CPS were shown as (a) the flat panel, (b) the cuboids, (c) the cylinders, and (d) the pyramids. In the same model, the top part is the surface deformation of the CPS, the bottom part is the partial detailed view of the microstructure. (e) The capacitance-pressure characteristics of the sensors with different microstructures. (f) The sensitivity and nonlinear error of the sensors with different microstructures. 


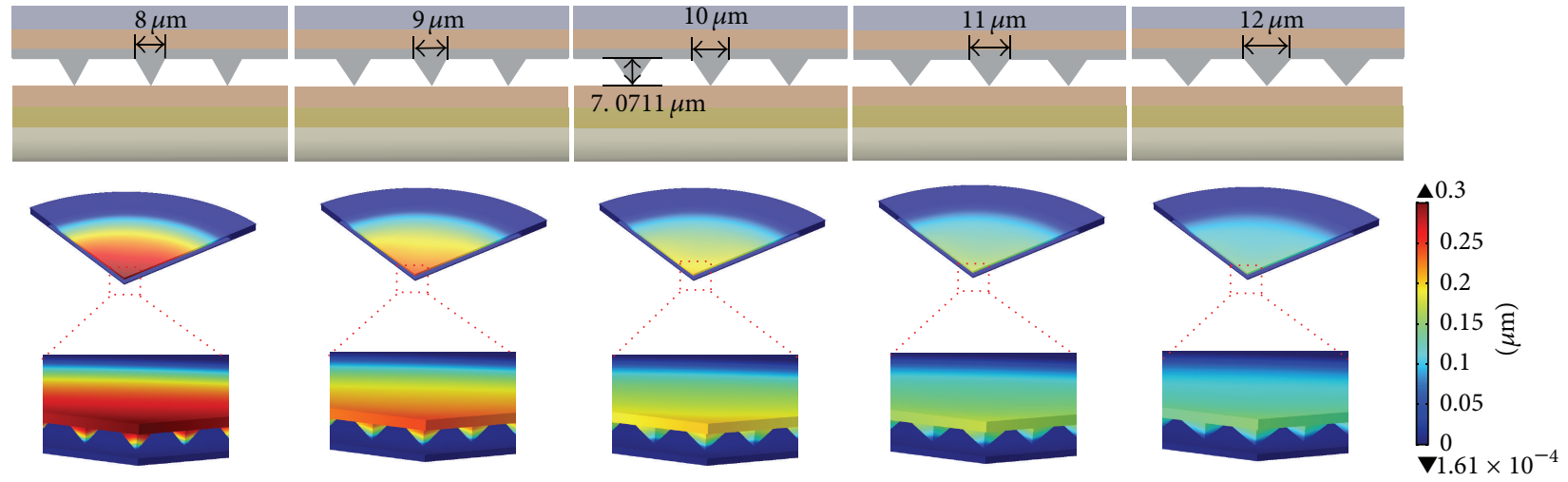

(a)

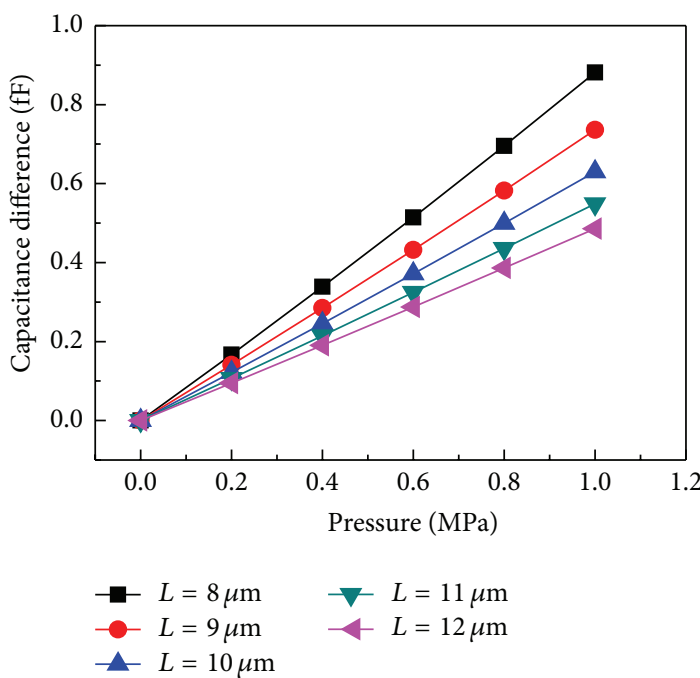

(b)

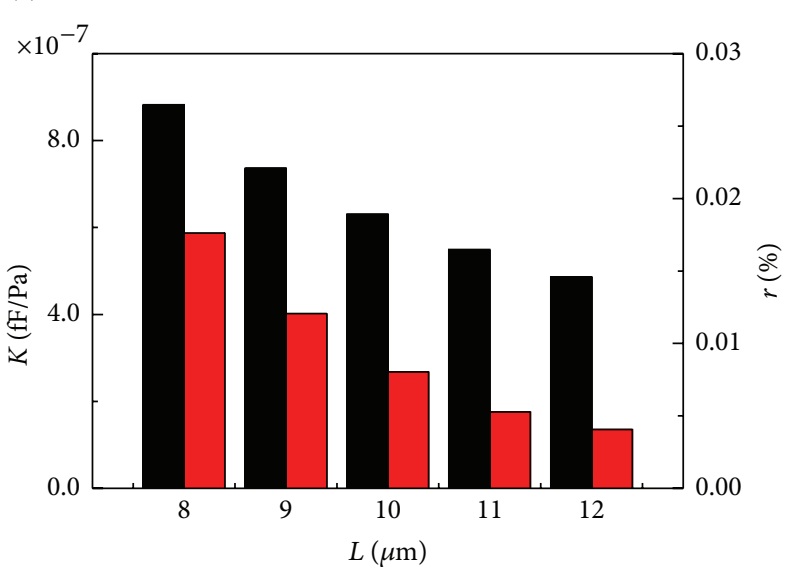

(c)

FIGURE 4: The pressure responses of the CPS with different lengths of hemline of pyramids. (a) The profile structures of sensors with different lengths of hemline of pyramid, from $8 \mu \mathrm{m}$ to $12 \mu \mathrm{m}$, and the same height $7.0711 \mu \mathrm{m}$, at $1 \mathrm{MPa}$. (b) The comparison of capacitance-pressure characteristics of the sensors with different lengths. (c) The sensitivity and nonlinear error of the sensors with different lengths.

TABLE 2: Sensitivity and nonlinear error of the CPS with different hemline lengths of pyramids.

\begin{tabular}{lcc}
\hline $\begin{array}{l}\text { Length of } \\
\text { hemline } L(\mu \mathrm{m})\end{array}$ & Sensitivity $K(\mathrm{fF} / \mathrm{Pa})$ & Nonlinear error $r$ \\
\hline 8 & $8.81355 E-7$ & $0.017621 \%$ \\
9 & $7.35721 E-7$ & $0.012068 \%$ \\
10 & $6.29792 E-7$ & $0.008034 \%$ \\
11 & $5.48525 E-7$ & $0.005271 \%$ \\
12 & $4.85630 E-7$ & $0.004066 \%$ \\
\hline
\end{tabular}

Figure 4(b) and the sensitivities and the nonlinear errors of CPS were shown in Figure 4(c) and Table 2.

From Table 2, both the sensitivity and the nonlinear error decrease with the increasing $L$. When hemline length $L$ and heights $H$ are $8 \mu \mathrm{m}$ and $7.07 \mu \mathrm{m}$, the sensor has the best sensitivity of $8.81 \times 10^{-7} \mathrm{fF} / \mathrm{Pa}$. In this case, in order to obtain greater sensitivity, we hope the hemline length is as small as possible. In other words, the smaller the ratios of $L / H$ of pyramid, the better the sensitivity of the CPS. But its nonlinear error would reach $0.018 \%$. As the change of ratios of $L / H$, we should balance the sensitivity of CPS for $e$-skin against the nonlinearity error to effectively tune the performance of CPS through choosing different parameters according to the required demands.

In analogy with the research between the pyramids' lengths of hemline and the sensor's performance, we have also made a series of simulations about the pyramids' heights. In the simulation, the height changes from $5 \mu \mathrm{m}$ to $9 \mu \mathrm{m}$ with the same length of hemline $(L=10 \mu \mathrm{m})$; the schematic diagram is shown in Figure 5(a).

From this schematic diagram, all the models have the same distance between the two electrodes; when the height of pyramids changes, the bottom of the pyramid will be filled with dielectric material. Likewise, the outside pressure changes from 0 to $1 \mathrm{MPa}$ too, the pressure responses of 


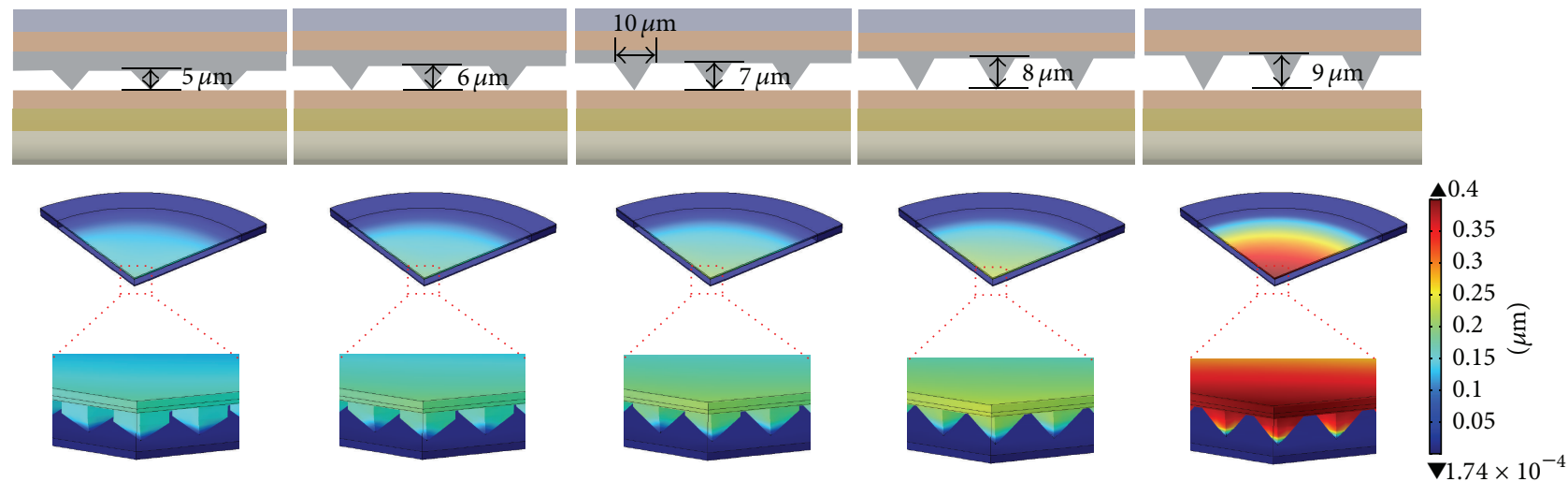

(a)

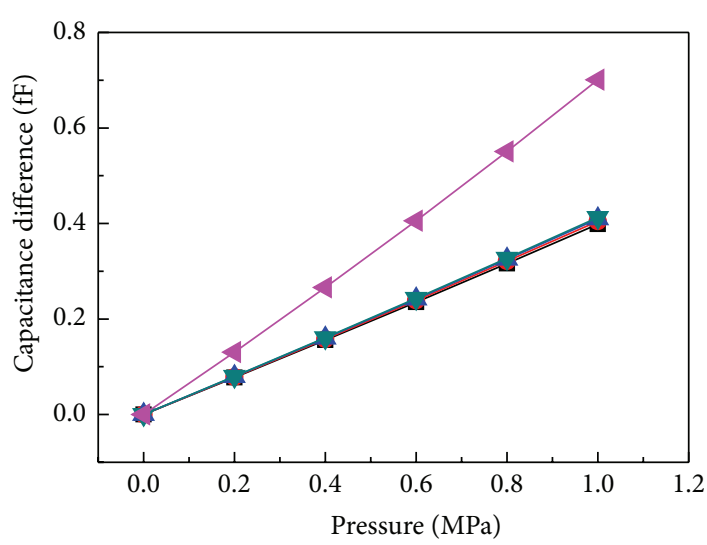

$-H=5 \mu \mathrm{m}$
$-H=6 \mu \mathrm{m}$
$-H=7 \mu \mathrm{m}$ $\neg H=8 \mu \mathrm{m}$

- $H=9 \mu \mathrm{m}$

(b)

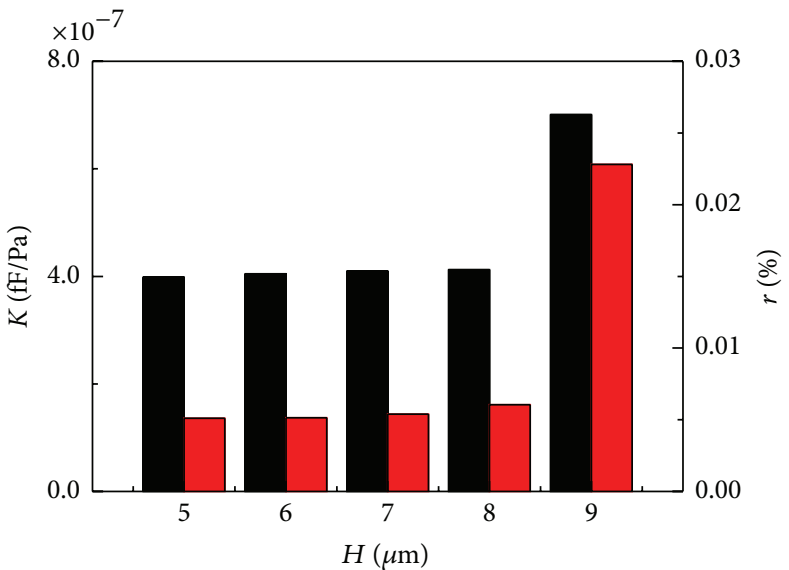

$K$

(c)

FIGURE 5: The pressure responses of the CPS with different heights of pyramids. (a) The profile structures of sensors with different heights of pyramid, from $5 \mu \mathrm{m}$ to $9 \mu \mathrm{m}$, and the same lengths of hemline $10 \mu \mathrm{m}$, at $1 \mathrm{MPa}$. (b) The comparison of capacitance-pressure characteristics of the sensors with different heights. (c) The sensitivity and nonlinear error of the sensors with different heights.

the sensor are obtained, and the capacitance-pressure curves of sensors are shown in Figure 5(b). The sensitivity and the linearity of sensors are shown in Figure 5(c) and Table 3.

From Figure 5 and Table 3, it is easy to find that the sensitivity increases with the height increases from $5 \mu \mathrm{m}$ to $9 \mu \mathrm{m}$, but the linearity is decreased. In the simulation of this series, when the length of hemline of pyramid is $10 \mu \mathrm{m}$ and the height of pyramid is $9 \mu \mathrm{m}$, the sensor has the best sensitivity, that is, $7.00589 \mathrm{E}-7 \mathrm{fF} / \mathrm{Pa}$. In this case, in order to obtain the greater sensitivity, we hope the height of pyramid is as large as possible. In other words, in order to obtain the greater sensitivity, we expect that the length of hemline of pyramid is more than $\sqrt{2}$ times the height.

From what has been discussed above, it is obvious that the performance of the sensor is affected by the length of hemline and the height simultaneously. Considering these two contradictory parameters, a compromise method is proposed to obtain the optimal performance of the sensor;
TABLE 3: Sensitivity and nonlinear error of the CPS with different heights of pyramids.

\begin{tabular}{lcc}
\hline Height $H(\mu \mathrm{m})$ & Sensitivity $K(\mathrm{fF} / \mathrm{Pa})$ & Nonlinear error $r$ \\
\hline 5 & $3.98334 E-7$ & $0.005111 \%$ \\
6 & $4.04683 E-7$ & $0.005135 \%$ \\
7 & $4.09844 E-7$ & $0.005384 \%$ \\
8 & $4.12467 E-7$ & $0.006055 \%$ \\
9 & $7.00589 E-7$ & $0.022813 \%$ \\
\hline
\end{tabular}

if the medians of $L$ and $H$ are selected, at this point, both the sensitivity and linearity have good values. Through the analysis of the relationship between $L$ and $H$, the author comes to the conclusion that in order to make the sensor have better performance the ratios of $L / H$ should be $\sqrt{2}$. 


\section{Conclusions}

In this paper, we employ the traditional COMSOL Multiphysics models based on finite element analysis method to construct four different microstructures (flat panel, cuboids, cylinders, and pyramids) CPS for the detailed study of microstructure interfacial tuning mechanism between human skin and PVDF. The simulative results show that CPS with micropyramids structures has the best measuring sensitivity $K\left(=6.3 \times 10^{-7} \mathrm{fF} / \mathrm{Pa}\right)$, which is about 49 times higher than that without any microstructure. Through the simulation of pyramids with different ratios of $L / H$, the results show that the smaller the ratios of $L / H$ of pyramid, the better the sensitivity, but the nonlinear error will increase. When the ratio of $L / H$ of pyramid is about $\sqrt{2}$, the sensitivity and the linearity could reach a balance point. This method can be used as theoretical guidance for the $e$-skin processing.

\section{Conflict of Interests}

The authors declare that there is no conflict of interests regarding the publication of this paper.

\section{Acknowledgments}

This work is supported by the National University Student Innovation Program (201510613009) of China, Sichuan Province Science and Technology Plan Project (no. 2015JQ0013), and the Fundamental Research Funds for the Central Universities (A0920502051408-10).

\section{References}

[1] M. L. Hammock, A. Chortos, B. C.-K. Tee, J. B.-H. Tok, and Z. Bao, "25th Anniversary article: the evolution of electronic skin (E-Skin): a brief history, design considerations, and recent progress," Advanced Materials, vol. 25, no. 42, pp. 5997-6038, 2013.

[2] T. W. Tombler, C. Zhou, L. Alexseyev et al., "Reversible electromechanical characteristics of carbon nanotubes under localprobe manipulation," Nature, vol. 405, no. 6788, pp. 769-772, 2000.

[3] B. Zhu, Z. Niu, H. Wang et al., "Microstructured graphene arrays for highly sensitive flexible tactile sensors," Small, vol. 10, no. 18, pp. 3625-3631, 2014.

[4] D. J. Cohen, D. Mitra, K. Peterson, and M. M. Maharbiz, "A highly elastic, capacitive strain gauge based on percolating nanotube networks," Nano Letters, vol. 12, no. 4, pp. 1821-1825, 2012.

[5] S. C. B. Mannsfeld, B. C.-K. Tee, R. M. Stoltenberg et al., "Highly sensitive flexible pressure sensors with microstructured rubber dielectric layers," Nature Materials, vol. 9, no. 10, pp. 859-864, 2010.

[6] W. Zhang and R.-G. Xiong, "Ferroelectric metal-organic frameworks," Chemical Reviews, vol. 112, no. 2, pp. 1163-1195, 2012.

[7] L. Persano, C. Dagdeviren, Y. Su et al., "High performance piezoelectric devices based on aligned arrays of nanofibers of poly(vinylidenefluoride-co-trifluoroethylene)," Nature Communications, vol. 4, article 1633, 2013.
[8] M. Ramuz, B. C.-K. Tee, J. B.-H. Tok, and Z. Bao, "Transparent, optical, pressure-sensitive artificial skin for large-area stretchable electronics," Advanced Materials, vol. 24, no. 24, pp. 32233227, 2012.

[9] R. Koeppe, P. Bartu, S. Bauer, and N. S. Saricifici, "Lightand touch-point localization using flexible large area organic photodiodes and elastomer waveguides," Advanced Materials, vol. 21, no. 34, pp. 3510-3514, 2009.

[10] R. Puers, "Capacitive sensors: when and how to use them," Sensors and Actuators A: Physical, vol. 37-38, pp. 93-105, 1993.

[11] J. A. Dobrzynska and M. A. M. Gijs, "Flexible polyimide-based force sensor," Sensors and Actuators A: Physical, vol. 173, no. 1, pp. 127-135, 2012.

[12] H. B. Muhammad, C. Recchiuto, C. M. Oddo et al., "A capacitive tactile sensor array for surface texture discrimination," Microelectronic Engineering, vol. 88, no. 8, pp. 1811-1813, 2011.

[13] C. S. Sander, J. W. Knutti, and J. D. Meindl, "A monolithic capacitive pressure sensor with pulse-period output," IEEE Transactions on Electron Devices, vol. 27, no. 5, pp. 927-930, 1980.

[14] H.-L. Chau and K. D. Wise, "An ultraminiature solid-state pressure sensor for a cardiovascular catheter," IEEE Transactions on Electron Devices, vol. 35, no. 12, pp. 2355-2362, 1988.

[15] S. S. Kumar and B. D. Pant, "Polysilicon thin film piezoresistive pressure microsensor: design, fabrication and characterization," Microsystem Technologies, vol. 21, no. 9, pp. 1949-1958, 2015.

[16] K. Takahata, Y. B. Gianchandani, and K. D. Wise, "Micromachined antenna stents and cuffs for monitoring intraluminal pressure and flow," Journal of Microelectromechanical Systems, vol. 15, no. 5, pp. 1289-1298, 2006.

[17] T. G. S. M. Rijks, P. G. Steeneken, J. T. M. van Beek et al., "Microelectromechanical tunable capacitors for reconfigurable RF architectures," Journal of Micromechanics and Microengineering, vol. 16, no. 3, pp. 601-611, 2006.

[18] K. I. Arshak, D. Morris, A. Arshak, O. Korostynska, and E. Jafer, "Development of a wireless pressure measurement system using interdigitated capacitors," IEEE Sensors Journal, vol. 7, no. 1, pp. 122-129, 2007.

[19] J. Ji, S. T. Cho, Y. Zhang, K. Najafi, and K. D. Wise, "An ultraminiature CMOS pressure sensor for a multiplexed cardiovascular catheter," IEEE Transactions on Electron Devices, vol. 39, no. 10, pp. 2260-2267, 1992.

[20] H. Tian, Y. Shu, X. Wang et al., "A graphene-based resistive pressure sensor with record-high sensitivity in a wide pressure range," Scientific Reports, vol. 5, article 8603, pp. 1-6, 2015.

[21] C. Tao, Z. Zhaohua, R. Tianling et al., "A novel dual-functional MEMS sensor integrating both pressure and temperature units," Journal of Semiconductors, vol. 31, no. 7, Article ID 074013, 2010.

[22] X. Huang and D. Zhang, "A high sensitivity and high linearity pressure sensor based on a peninsula-structured diaphragm for low-pressure ranges," Sensors and Actuators A: Physical, vol. 216, pp. 176-189, 2014.

[23] N. J. Madduri, G. Lakkoju, B. L. Kasturi, S. Sravanam, and T. Satyanarayana, "Design and deformation analysis of MEMS based piezoresistive pressure sensor," International Journal of Automotive Engineering and Technologies, vol. 7, pp. 521-531, 2014.

[24] B. A. Ganji and M. Shahiri-Tabarestani, "A novel high sensitive MEMS intraocular capacitive pressure sensor," Microsystem Technologies, vol. 19, no. 2, pp. 187-194, 2013. 
[25] K. Hosoda, Y. Tada, and M. Asada, "Anthropomorphic robotic soft fingertip with randomly distributed receptors," Robotics and Autonomous Systems, vol. 54, no. 2, pp. 104-109, 2006.

[26] J. C. Helton, J. D. Johnson, C. J. Sallaberry, and C. B. Storlie, "Survey of sampling-based methods for uncertainty and sensitivity analysis," Reliability Engineering and System Safety, vol. 91, no. 10-11, pp. 1175-1209, 2006.

[27] J. Y. Zhao, Detection and Sensing Technology, Southwest Jiaotong University Press, Chengdu, China, 2007.

[28] L. Lin, H.-C. Chu, and Y.-W. Lu, "Simulation program for the sensitivity and linearity of piezoresistive pressure sensors," Journal of Microelectromechanical Systems, vol. 8, no. 4, pp. 514522, 1999. 


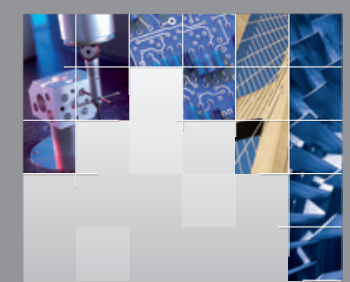

\section{Enfincering}
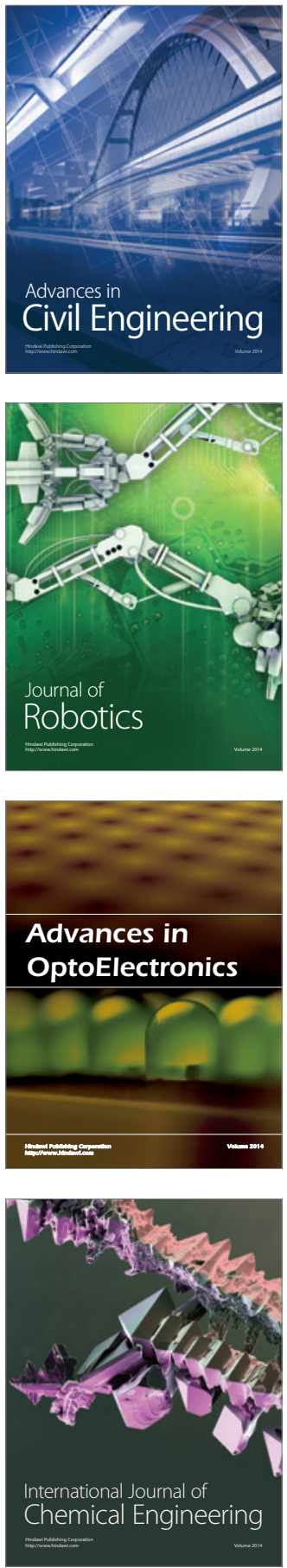

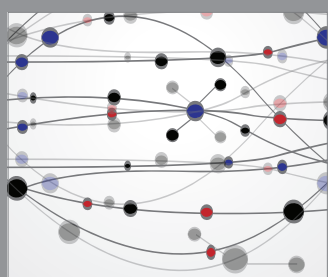

The Scientific World Journal

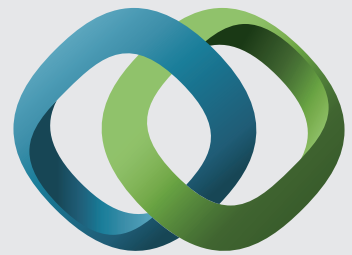

\section{Hindawi}

Submit your manuscripts at

http://www.hindawi.com
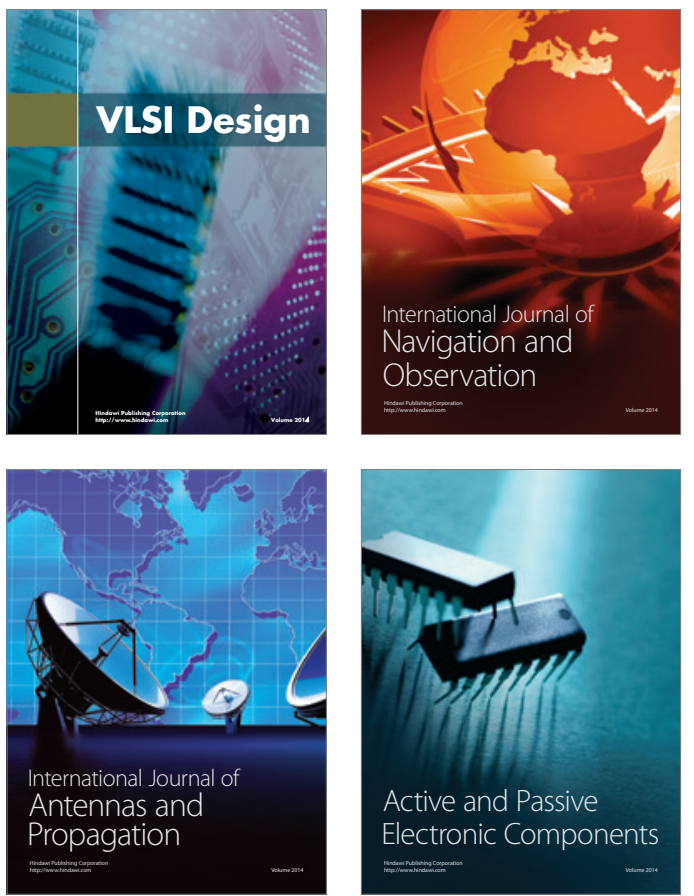
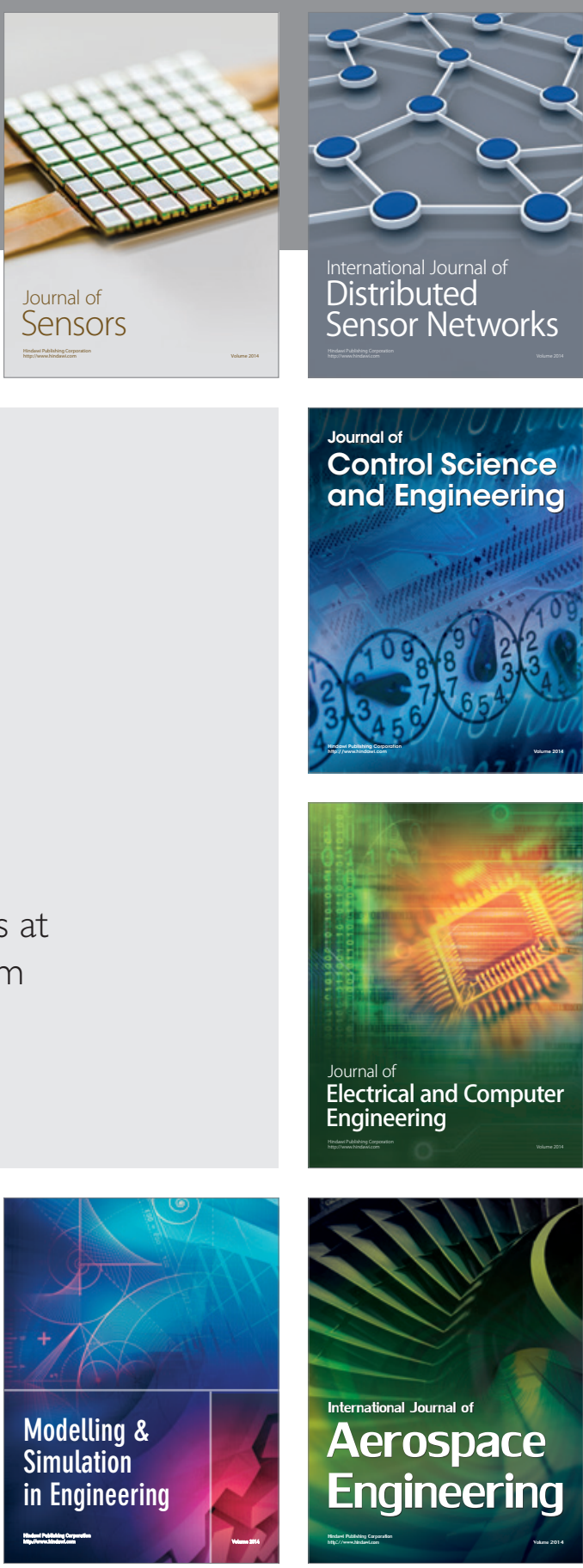

International Journal of

Distributed

Sensor Networks

Journal of

Control Science

and Engineering
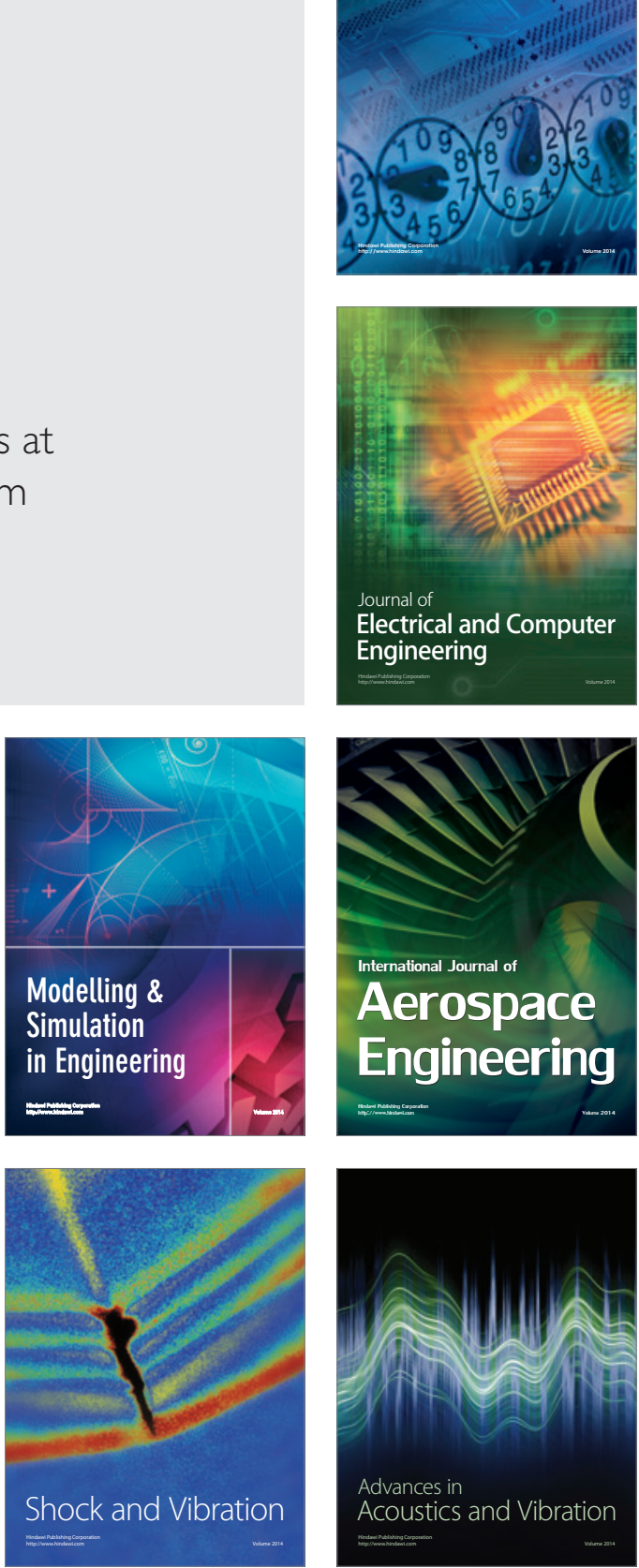